\title{
Theoretical Analysis of Non-ablative Laser Texturing of Silicon Surface with a Continuous Wave Fiber Laser
}

\author{
Hamid Farrokhi ${ }^{* 1}$, Wei Zhou ${ }^{* 1,2}$ and Hongyu Zheng ${ }^{* 3}$ \\ ${ }^{*}$ School of Mechanical and Aerospace Engineering, Nanyang Technological University, 50Nanyang \\ Avenue, Singapore 639798 \\ E-mail: hami0006@e.ntu.edu.sg \\ ${ }^{* 2}$ College of Materials Science and Engineering, Hefei University of Technology, Hefei 230009, P. \\ R. China \\ ${ }^{* 3}$ Machining Technology Group, Singapore Institute of Manufacturing Technology, 71 Nanyang \\ Drive, Singapore 638075
}

\begin{abstract}
$\mathrm{Si}$ is highly transparent to infrared fiber laser $(\lambda=1090 \mathrm{~nm})$ and the laser is not regarded as a suitable tool for ablation based silicon surface texturing. However, we have shown non-ablative texturing of Si surface is possible provided that suitable laser power and dwell time are chosen to produce peak surface temperature above the Si effective oxidation point of $1250 \mathrm{~K}$ and below the $\mathrm{Si}$ melting point of $1690 \mathrm{~K}$. The phenomenon is explained through theoretical thermal analysis. The Kirchhoff transform is used to include the temperature dependent thermal conductivity. It is shown that with carefully adjusting laser parameters localized oxidation occurs and results in the formation of regularly patterned micro-bumps.
\end{abstract}

DOI: $10.2961 / \mathrm{jlmn} .2015 .02 .0014$

Keywords: Non-ablative micro/nano texturing; thermal analysis; laser-induced oxidation

\section{INTIRUCTION}

Surface texturing became known as an important field of research with a broad range of applications in electronics, solar cells, and sensors. Focused ion beam (FIB), for instance, has been used to produce micro- and nano-scale surface patterns either by direct FIB writing [1-3] or through ion beam induced self-organization [4-6]. However, this type of patterning has to be conducted in high vacuum for very small surface areas. In contrast, laser beam can be used to pattern surface in ambient or controlled environments over large surface areas [7-9].

$\mathrm{Si}$ is an important semiconductor material, so texturing its surface by laser has been well studied in the past [10-14]. However, all the surface texturing is based upon ablation. Hardly any report can be found on use of non-ablative laser surface texturing to form uniform micro-patterns. Recently, we succussed to pattern $\mathrm{Si}$ surface by non-ablative laser texturing using continuous wave fiber laser $(\lambda=1090 \mathrm{~nm})$ [15-17]. As $\mathrm{Si}$ is known to be highly transparent to the infrared wavelength, the fiber laser is not regarded as a suitable tool for texturing of Si surface. We elucidated the non-ablative surface texturing in terms of laser-induced localized thermal oxidation $[15,16]$. However, the current study aimed to gain better understanding of the nonablative texturing mechanism through theoretical thermal analysis and to provide a quick-estimation tool for determining the suitable laser parameters for the surface texturing. The non-ablative texturing method provides possibilities for micro/nano-device applications, clean and free-crack marking of silicon wafers.

\section{EXPERIMENATL PROCEDURE AND RESULTS}

The material used in the study was a single crystal (100) n-type (phosphorous doped) Si wafer with resistivity $\rho=1-20 \Omega \cdot \mathrm{cm}$ and thickness of $300 \mu \mathrm{m}$. The wafer was cut into smaller pieces in the size of $10 \mathrm{~mm} \times 10 \mathrm{~mm}$. Ultrasonic cleaning of the samples was carried out in methanol for $10 \mathrm{~min}$, acetone for another $10 \mathrm{~min}$, and then de-ionized water for $30 \mathrm{~min}$. To minimize influence of native oxide layer, the cleaned samples were kept in deionized water till being used.

The samples were mounted on a motorized X-Y precision stage and irradiated using a continuous wave fibre laser with wavelength $\lambda=1090 \mathrm{~nm}$. The laser scanning was carried out in an area of $2 \mathrm{~mm} \times 2 \mathrm{~mm}$ with a point scanning pitch of $20 \mu \mathrm{m}$, as shown in Figure 1. A lens of $50 \mathrm{~mm}$ focal length was used to focus the laser beam to the size of $\approx 20 \mu \mathrm{m}$.

The laser experiments were carried out using varying laser power $(11.5,19$, or $23.3 \mathrm{~W})$ and dwell time $(10,20$, 30 , or $40 \mathrm{~ms}$ ) in $\mathrm{O}_{2}$ atmosphere. $\mathrm{O}_{2}$ was delivered onto the $\mathrm{Si}$ surface in the pressure of 0.5 bar through a coaxial nozzle with an exit diameter of $2 \mathrm{~mm}$ and placed about 2 $\mathrm{mm}$ above the surface, as shown in Figure 1.

Surfaces irradiated with the fiber laser were carefully observed under the confocal microscope and the 3D optical profilometer. We summarize our observation as follows:

(1) No discernible change was found on surface irradiated using the lowest power of $11.5 \mathrm{~W}$.

(2) Considerable morphological changes were found on surface irradiated with the highest power of $23.3 \mathrm{~W}$, but the surface patterns were irregular, as shown in Figure 2(a).

(3) When the moderate power of $19 \mathrm{~W}$ was used, regularly patterned surface features were formed when the dwell time was $40 \mathrm{~ms}$, as shown in Figure 2(b); however, no such uniform surface patterns were observed for the 
lower dwell times $(10,20$, or $30 \mathrm{~ms})$. The regularly patterned surface was further studied using 3D optical profilometer, as shown in Figures 3(a) and 3(b).

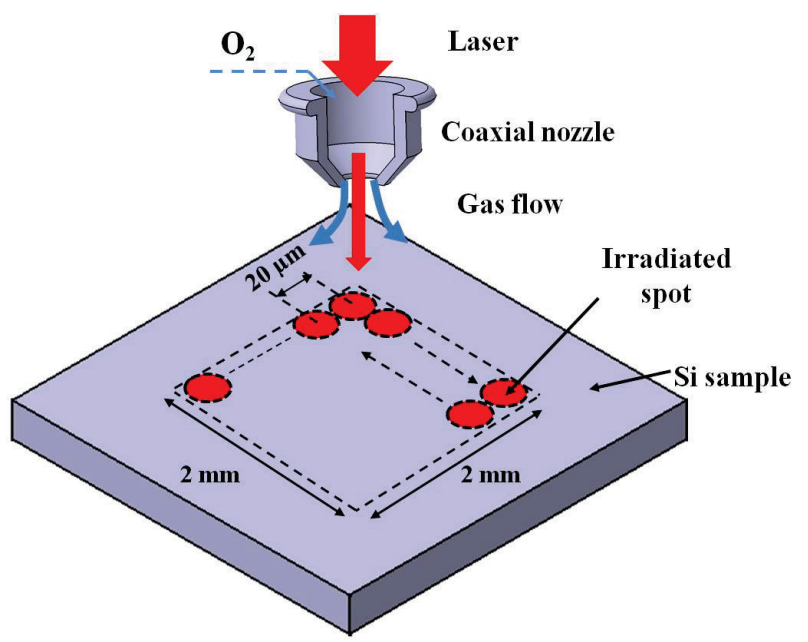

Fig. 1 Illustration of experimental setup
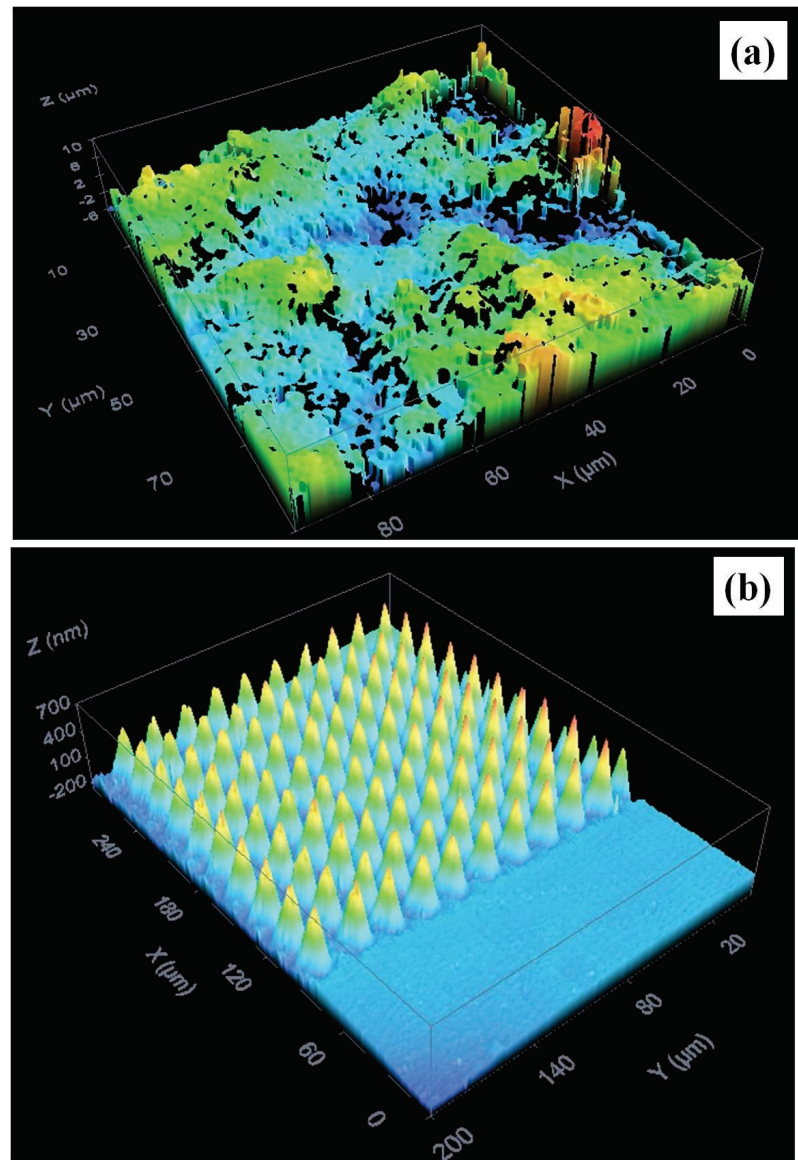

Fig. 2 Confocal microscope images of surface irradiated with (a) laser power of $23.3 \mathrm{~W}$, dwell time of $40 \mathrm{~ms}$ and (b) laser power of $19 \mathrm{~W}$, dwell time of $40 \mathrm{~ms}$.

Figure 3(a) shows 3D image of the uniformly patterned surface irradiated with laser power $=19 \mathrm{~W}$ and dwell time $=40 \mathrm{~ms}$. Figure $3(\mathrm{~b})$ demonstrates $2 \mathrm{D}$ profile of a bump with height of $\approx 750 \mathrm{~nm}$ and base diameter of about $13 \mu \mathrm{m}$. More detailed description of the experimental data could be found in [15].
In the following sections, we will attempt to elucidate the phenomena by theoretical thermal analysis.
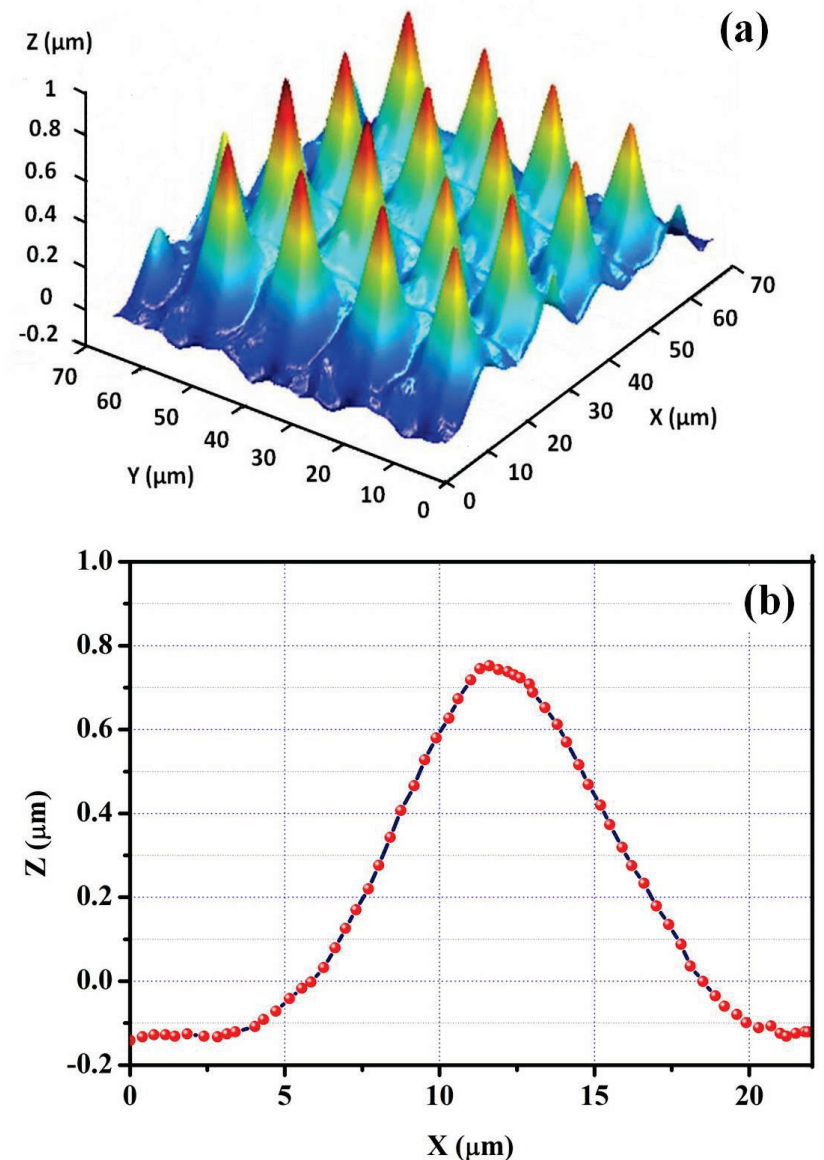

Fig. 3 (a) 3D optical image of surface irradiated with laser power: $19 \mathrm{~W}$ and dwell time: $40 \mathrm{~ms}$. (b) $2 \mathrm{D}$ profile of a bump shown in (a).

\section{Theoretical Analysis}

3.1. Kirchhoff transform - linear and true temperature

The familiar heat transfer equation for the temperature, $\mathrm{T}$, is the diffusion equation:

$$
\rho C_{p}(T) \frac{\partial T}{\partial t}=\nabla[k(T) \nabla T]+Q(r, t)
$$

where $k$ is the thermal conductivity, $\rho$ the density, and $C_{p}$ the specific heat of the material, and $Q(r, t)$ is rate at which energy is being supplied to the material. The density and specific heat can be assumed to be constant for $\mathrm{Si}$, but the thermal conductivity varies approximately as $1 / \mathrm{T}$ above room temperature. Thus equation (1) is strongly nonlinear.

The nonlinear heat flow equation can be simplified by introducing the Kirchhoff transformation [18]:

$$
\theta(t)=\int_{T_{0}}^{T(t)} \frac{k\left(T^{\prime}\right)}{k_{0}} d T^{\prime}
$$

where $k_{0}$ is the thermal conductivity at a reference temperature $T_{0}$, which is chosen to be the initial substrate temperature. 
The linear temperature rise, $\theta(t)$, is then related to the true temperature rise, $\mathrm{T}(\mathrm{t})$, by equation (2). In the steadystate condition, the equation for $\theta$ is the linear heat flow equation, and therefore we name $\theta$ the linear temperature. The temperature distribution for the steady-state can thus be obtained by first solving the linear heat flow equation for $\theta$, with constant conductivity $k_{0}$. The true temperature $\mathrm{T}$ is then calculated by using the inverse of Kirchhoff transform $[19,20]$.

The thermal conductivity of $\mathrm{Si}$ can be adequately approximated using equation (3)[20].

$$
k_{T}=A /(T-B)
$$

where $\mathrm{A}=299 \mathrm{~W} / \mathrm{cm}$ and $\mathrm{B}=99 \mathrm{~K}$. Thus, the inverse of Kirchhoff transform can be expressed analytically as below:

$$
T=B+\left(T_{0}-B\right) \exp \left[\theta(\mathrm{t}) /\left(\mathrm{T}_{0}-\mathrm{B}\right)\right]
$$

Spatial intensity distribution of the laser is considered to be :

$$
I=\left(\mathrm{P}_{a b s} / \pi w^{2}\right) \exp \left(-r^{2} / w^{2}\right)
$$

where $r$ is the distance from the center of the beam and $P_{a b s}$ is the total absorbed power. $w$ is beam radius at the $1 / \mathrm{e}$ of the peak power.

The beam is centered on the point of interest, $(x, y)=(0,0)$, at time $t=0$. The maximum linear temperature rise at $\mathrm{t}=0$ is derived as below.

$$
\theta_{\max }=\frac{P_{a b s}}{2 \sqrt{\pi} w k_{0}}
$$

The results of equation (6) can now be inserted into equation (4) to calculate the maximum true temperature rise.

$$
T_{\max }=B+\left(T_{0}-B\right) \exp \left[\frac{P_{a b s}}{2 \sqrt{\pi} w A}\right]
$$

Noted that the equation (7) is derived to calculate the maximum surface temperature rise in the steady-state condition. Thus, in the next section we have specified the condition.

\subsection{Temperature versus time for constant intensity}

The condition in which the steady-state solution for solving the heat equation can be used is determined using numerical method.

Because of the nonlinearity of equation (1), we have chosen the three time level implicit method for numerical solution of the temperature. The thermal conductivity is given by equation ( 3 ). We use $\rho=2.3 \mathrm{~g} / \mathrm{cm}^{3}$ and $C_{p}=0.88$ $\mathrm{J} / \mathrm{gK}$ for the density and specific heat of $\mathrm{Si}$. For simplicity we assume a single absorption coefficient, $\alpha=501 / \mathrm{cm}$. The value of the absorption coefficient has little effect on how quickly the equilibrium is achieved.
Figure 4 shows the results of numerical method for a $300 \mu \mathrm{m}$ thick sample subjected to incident power of 23.3 , 19 , and $11.5 \mathrm{~W}$ with $\mathrm{T}_{0}=298 \mathrm{~K}$. It is shown that $98 \%$ of the final temperature rise is reached within $10 \mathrm{~ms}$, and after 15 ms the sample is in relative steady-state. This means that the steady-state solution for the temperature can be reasonably used if half of the irradiation dwell time is more than $15 \mathrm{~ms}$. It implies that the steady-state solution could be valid for the dwell time above $30 \mathrm{~ms}$.

Since the condition for the steady-state will normally be met in experiments with $\mathrm{CW}$ fiber laser and dwell time of $40 \mathrm{~ms}$, we will limit the study to the time-independent case..

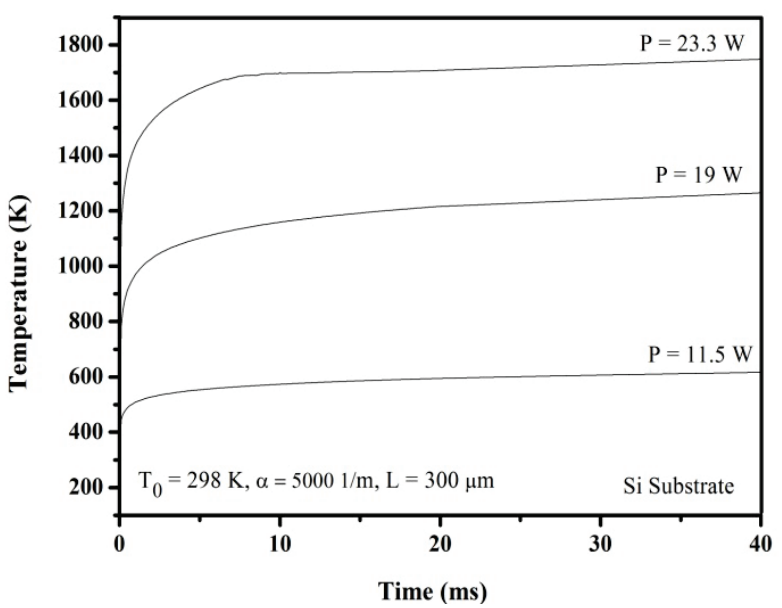

Fig. 4 Surface temperature vs time of a Si sample irradiated with a constant incident power of $23.3,19,11.5 \mathrm{~W}$. The absorption coefficient $\alpha$ is $50001 / \mathrm{m}$; and sample thickness is $300 \mu \mathrm{m}$. The results are obtained numerically.

\subsection{Temperature rise in the steady-state condition}

We have demonstrated that the steady-state solution is applicable for $\mathrm{CW}$ irradiation with the dwell time above $30 \mathrm{~ms}$. Equation (7) is used to obtain the maximum surface temperature for various incident powers $(11.5,19,23.3 \mathrm{~W})$ at fixed dwell time of $40 \mathrm{~ms}$. The results are shown in Table1.

Table1 Maximum surface temperature at various incident laser power.

\begin{tabular}{cccc}
\hline $\begin{array}{c}\text { Incident laser } \\
\text { power } \\
(\mathbf{W})\end{array}$ & 11.5 & 19 & 23.3 \\
\hline $\begin{array}{c}\mathbf{T}_{\max }(\mathbf{K}) \\
\text { (steady-state) }\end{array}$ & 688 & 1294 & 1892 \\
\hline $\begin{array}{c}\mathbf{T}_{\max }(\mathbf{K}) \\
(\text { Numerical) }\end{array}$ & 610 & 1265 & 1760 \\
\hline $\begin{array}{c}\mathbf{T}_{\max }(\mathbf{K}) \\
(\mathrm{FE})[16]\end{array}$ & 619 & 1304 & 1840 \\
\hline Dwell time $=40 \mathrm{~ms}, \mathrm{R}=0.33, \mathrm{~T}=0.51$ \\
\hline
\end{tabular}

To obtain the absorbed power, $\mathrm{P}_{\mathrm{abs}}$, the following equation has been used.

$$
P_{a b s}=(1-R-T) P_{i n c}
$$


where $\mathrm{P}_{\text {inc }}$ is incident laser power, $\mathrm{R}$ and $\mathrm{T}$ are reflection and transmission coefficient of $\mathrm{Si}$ at the fiber laser wavelength $(1090 \mathrm{~nm})$.

It is shown that for dwell times above $30 \mathrm{~ms}$, the surface temperature rise obtained through steady-state solution is in fair agreement with that of obtained through numerical method and finite element (FE) analysis [16] . It suggests that the steady-state solution could be quick-estimation tool for determining the suitable laser parameters for nonablative surface texturing comparing with time-consuming numerical method.

\section{Discussion}

Results of the maximum surface temperature at center of the irradiated point are shown in Table 1. For the highest laser power of $20.3 \mathrm{~W}$, It shows that the surface temperature is above the melting point of $\mathrm{Si}(1690 \mathrm{~K})$. . This explain the formation of irregular surface patterns when the surface is irradiated with this laser power (see Figure 2(a)). The result is in good agreement with that of obtained through FE study [16].

It can be found from Table 1 that no melting would occur on surfaces irradiated with laser power of $11.5 \mathrm{~W}$ and $19 \mathrm{~W}$ for dwell time $=40 \mathrm{~ms}$, since the surface temperature is below the melting point of $\mathrm{Si}$. It shows good agreement with our previous study carried out using FE analysis [16].

If localized thermal oxidation of $\mathrm{Si}$ accounts for formation of the regular bumps as suggested earlier [15], regular bumps might be expected to form for laser power of both $11.5 \mathrm{~W}$ and $19 \mathrm{~W}$. However, in reality, regular surface bumps were formed only when the laser power was $19 \mathrm{~W}$ and the dwell time was $40 \mathrm{~ms}$. The thermal analysis results may help to explain why this is the case.

It is found in many studies [21-25] that thermal oxidation of Si surface generally occurs in the temperature range $1000-1600 \mathrm{~K}$ and that $56 \%$ of the oxide thickness rises above the original silicon surface. For the laser power of $11.5 \mathrm{~W}$, the steady-state solution, numerical method and FE study [16] showed the peak temperature is well below $1000 \mathrm{~K}$ (see Table 1). Therefore, it is understandable why no surface bumps were formed due to thermal oxidation for this laser power.

For laser power of $19 \mathrm{~W}$, numerical method (see Fig.4) and FE study [16] showed the peak temperature is above $1000 \mathrm{~K}$ for all the dwell times used (10, 20, 30, and $40 \mathrm{~ms})$. In addition, steady-state solution which is valid for dwell time above $30 \mathrm{~ms}$ shows the same finding for the dwell time of $40 \mathrm{~ms}$. However, it is noticed that regular bumps formed only when the longest dwell time (40 ms) was used. This interesting phenomenon is believed to be related to decrease of activation energy of $\mathrm{Si}$ oxidation with increasing temperature.

Activation energy of $\mathrm{Si}$ oxidation lies around $2-2.5 \mathrm{eV}$ for temperature range 1100 to $1200 \mathrm{~K}$, but the value decreases to $1.3 \mathrm{eV}$ when the temperature reaches $1250 \mathrm{~K}$ [23]. In the current study, an infrared laser with wavelength $1090 \mathrm{~nm}$ was used. Photon energy for this laser is calculated as $E_{\text {photon }}=1.138 \mathrm{eV}$. When the temperature is below $1250 \mathrm{~K}$, the photon energy is well below the activation energy of $\mathrm{Si}$ oxidation (which is $2-2.5 \mathrm{eV}$ for temperature range $1100-1200 \mathrm{~K}$ and $1.3-2 \mathrm{eV}$ for temperature range 1200-1250 K [23]), making it difficult for effective $\mathrm{Si}$ oxidation to occur at temperatures below $1250 \mathrm{~K}$. When the laser power is $19 \mathrm{~W}$ and dwell time is $10 \mathrm{~ms}$ or $20 \mathrm{~ms}$, numerical method and FE study [16] showed the peak temperature is below $1250 \mathrm{~K}$, so no effective Si oxidation occurs.

When the temperature rises to $1250 \mathrm{~K}$, the activation energy of Si oxidation is reduced to $1.3 \mathrm{eV}$, which is very close to but still above the value of photon energy (1.138 $\mathrm{eV})$. When the temperature rises further, the activation energy reduces further. Therefore, at a certain temperature above $1250 \mathrm{~K}$, the activation energy falls below the photon energy, making it possible for the effective oxidation of $\mathrm{Si}$ to occur and resulting in localized surface expansion and bump formation.

For laser power of $19 \mathrm{~W}$ and dwell time of $30 \mathrm{~ms}$ numerical method and FE study [16] showed that the peak temperature is just above $1250 \mathrm{~K}$. The fact that no bumps were observed on the laser irradiated surface suggests that the activation energy at this peak temperature is not yet reduced to below the photon energy level.

When the dwell time is increased to $40 \mathrm{~ms}$, steady-state solution as well as numerical method and FE study [16] shows the peak temperature increases well above effective oxidation temperature $(1250 \mathrm{~K})$. It is shown to be $1294 \mathrm{~K}$ obtained by steady-state solution (see Table 1). The fact that bumps were observed on the laser irradiated surface indicates that the activation energy at $1294 \mathrm{~K}$ is reduced to below the photon energy. It can be deduced from the analyses that the critical temperature for effective oxidation of $\mathrm{Si}$ is somewhere between 1250 and $1300 \mathrm{~K}$. The temperature of $1250 \mathrm{~K}$ is used as the lower boundary for effective oxidation, and the upper boundary is naturally the melting point $(1690 \mathrm{~K})$.

Furthermore, steady-state solution might be quickestimation tool to predict a range of laser powers suitable for making oxide bumps through the non-ablative method. Figure 5 shows various incident laser powers at dwell time of $40 \mathrm{~ms}$ which can be used for the purpose of surface texturing.

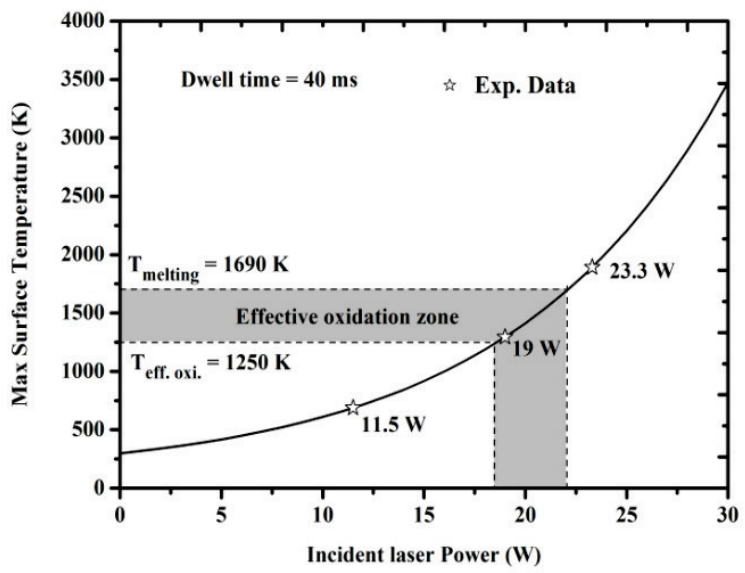

Fig. 5 Maximum surface temperature vs incident laser power obtained through steady-state thermal analysis, showing the effective oxidation zone to produce regular bumps on Si surface. 
Estimated power range gives the maximum surface temperature which falls between the two temperature boundary lines named as effective oxidation zone.

\section{Conclusions}

A regular array of micro-bumps was fabricated on $\mathrm{Si}$ surface using continuous wave fiber laser $(\lambda=1090 \mathrm{~nm})$ based on laser induced non-ablative texturing. Steady-state thermal analysis was used to explain the formation phenomenon. The condition for using the steady-state solution was obtained by numerical method. It is found that the bumps formation is due to laser-induced localized $\mathrm{Si}$ oxidation. A range of laser powers at the fixed dwell time of $40 \mathrm{~ms}$, suitable for the non-ablative surface texturing, was determined using theoretical results and it is named as the effective oxidation zone. The solution might be used as a quick-estimation tool to specify the suitable laser power to produce uniformly patterned surface features on $\mathrm{Si}$.

\section{References}

[1] H. X. Qian, Z. Wei, M. Jianmin, E. N. L. Lennie, and X. R. Zeng: J. Micromech. Microeng., 18, (2008) 035003. [2] Y. Fu, N. Bryan, and W. Zhou: Opt. Express, 12, (2004) 1803.

[3] Y. Fu, W. Zhou, L. E. N. Lim, C. Du, X. Luo, Z. Zhao, X. Dong, H. Shi, and C. Wang: Opt Eng, 45, (2006) 108001

[4] J. Lian, W. Zhou, Q. M. Wei, L. M. Wang, L. A. Boatner, and R. C. Ewing: Appl. Phys. Lett., 88, (2006) 093112

[5] H. X. Qian, W. Zhou, Y. Q. Fu, B. K. A. Ngoi, and G. C. Lim: Appl. Surf. Sci., 240, (2005) 140.

[6] H. X. Qian, and W. Zhou: Mater. Lett. , 77, (2012) 113.

[7] H. Y. Zheng, W. Zhou, H. X. Qian, T. T. Tan, and G. C. Lim: Appl. Surf. Sci. , 236, (2004) 114.

[8] H. X. Qian, W. Zhou, H. Y. Zheng, and G. C. Lim: Surf. Sci., 595, (2005) 49.

[9] Z. L. Li, T. Liu, C. C. Khin, A. C. Tan, L. E. Khoong, H. Y. Zheng, and W. Zhou: Opt. Express, 18, (2010) 15990.

[10] T.-H. Her, R. J. Finlay, C. Wu, S. Deliwala, and E. Mazur: Appl. Phys. Lett., 73, (1998) 1673.

[11] V. Zorba, I. Alexandrou, I. Zergioti, A. Manousaki, C. Ducati, A. Neumeister, C. Fotakis, and G. A. J. Amaratunga: Thin Solid Films, 453-454, (2004) 492.

[12] V. Zorba, P. Tzanetakis, C. Fotakis, E. Spanakis, E. Stratakis, D. G. Papazoglou, and I. Zergioti: Appl. Phys. Lett., 88, (2006) 081103.

[13] P. Simon, and J. Ihlemann: Appl. Surf. Sci., 109110 , (1997) 25.

[14] B. Borchers, J. Bekesi, P. Simon, and J. Ihlemann: J. Appl. Phys., 107, (2010) 063106.

[15] H. Farrokhi, W. Zhou, H. Y. Zheng, and Z. L. Li: Opt. Express, 20, (2012) 23180.

[16] H. Farrokhi, w. Zhou, H. Zheng, and Z. Li: J. Comput. Theor. Nanosci., 11, (2014) 1.

[17] H. Farrokhi, W. Zhou, H. Y. Zheng, and Z. Li: Proc. of SPIE Vol. 7926, p. 792607.

[18] H. S. Carslaw, and J. C. Jeager: "Conduction of Heat in Solids", (Clarendon Press, Oxford 1986).
[19] M. Lax: J. Appl. Phys., 48, (1977) 3919.

[20] Y. I. Nissim, A. Lietoila, R. B. Gold, and J. F. Gibbons: J. Appl. Phys., 51, (1980) 274.

[21] J. Blanc: Appl. Phys. Lett., 33, (1978) 424.

[22] V. K. Samalam: Appl. Phys. Lett., 47, (1985) 736.

[23] A. Fargeix, and G. Ghibaudo: J. Appl. Phys., 56, (1984) 589.

[24] H. Z. Massoud, J. D. Plummer, and J. D. Irene: J. Electrochem. Soc, 132, (1985) 2693.

[25] B. E. Deal, and A. S. Grove: J. Appl. Phys., 36, (1965) 3770.

(Received: September 19, 2014, Accepted: March 3, 2015) 